\title{
The significance of podoplanin expression in human inflamed gingiva
}

\author{
Yuji Miyazaki ${ }^{1)}$, Eri Okamoto ${ }^{2)}$, Patricia González-Alva ${ }^{1)}$, Joichiro Hayashi ${ }^{3)}$, \\ Toshiyuki Ishige ${ }^{4)}$, Kentaro Kikuchi ${ }^{1,4)}$, Norimichi Nemoto ${ }^{4)}$, Kitetsu Shin ${ }^{3}$, \\ Hideaki Sakashita $^{2)}$, Kuniyasu Ochiai' ${ }^{5)}$ and Kaoru Kusama1) \\ ${ }^{1)}$ Division of Pathology, Department of Diagnostic and Therapeutic Sciences, Meikai University \\ School of Dentistry, Saitama, Japan \\ ${ }^{2)}$ Second Division of Oral and Maxillofacial Surgery, Department of Diagnostic and Therapeutic Sciences, \\ Meikai University School of Dentistry, Saitama, Japan \\ ${ }^{3}$ Division of Periodontology, Department of Oral Biology and Tissue Engineering, Meikai University \\ School of Dentistry, Saitama, Japan \\ ${ }^{4)}$ Department of Pathology, Nihon University School of Medicine, Tokyo, Japan \\ 5) Department of Microbiology, Nihon University School of Dentistry, Tokyo, Japan
}

(Received 20 February and accepted 23 March 2009)

\begin{abstract}
Podoplanin, a transmembrane glycoprotein, has been considered to be expressed specifically by lymphatic endothelial cells. However, recent studies have shown that the protein is expressed in a variety of normal as well as neoplastic tissues, and that its expression might be related to cell migration and invasion. In this study, we examined podoplanin expression in inflamed gingival tissues using an immunohistochemical method. Positive immunoreactivity for podoplanin was found in the cell membrane and cytoplasm of basal cells of oral gingival epithelium when severe inflammatory cell infiltration was present in the connective tissue just under the epithelium. When inflammatory changes were weak or absent, little or no reactivity for podoplanin in the basal cells was observed. Positive reactivity for podoplanin was also detected in basal cell extensions. Surprisingly, strong immunoreactivity for podoplanin was observed in all layers of oral sulcular and junctional
\end{abstract}

Correspondence to Dr. Kaoru Kusama, Division of Pathology, Department of Diagnostic and Therapeutic Sciences, Meikai University School of Dentistry, 1-1 Keyakidai, Sakado, Saitama 350-0248, Japan

Tel: +81-49-279-2773

Fax: +81-49-286-6101

E-mail: kusama@dent.meikai.ac.jp epithelia associated with severe inflammatory reaction in the connective tissue. These findings suggest that increased expression of podoplanin in gingival epithelium is related to the progression of chronic periodontitis. (J Oral Sci 51, 283-287, 2009)

Keywords: periodontitis; podoplanin; immunohistochemistry.

\section{Introduction}

Chronic periodontitis, an inflammatory form of periodontal disease, is caused by microorganisms present in the plaque biofilm that forms around the teeth (1). The formation of periodontal pockets, and progressive destruction of connective tissue and alveolar bone result in tooth loss in adults (1). The infiltration and extension of the junctional epithelium along the root caused by inflammatory stimuli from periodontopathic bacteria are essential events for the progression of chronic periodontitis (1). Podoplanin, a transmembrane glycoprotein, is expressed specifically by lymphatic endothelial cells $(2,3)$. However, recent studies have shown that the protein is expressed in a variety of normal as well as neoplastic tissues, and that its expression might be related to cell migration and invasion (4-6). In the present study, we 
examined whether podoplanin, a transmembrane glycoprotein, is related to the progression of chronic periodontitis, by analyzing the immunohistochemical expression of podoplanin in inflamed gingival tissues.

\section{Materials and Methods \\ Collection of samples}

Forty inflamed gingival tissue samples from the Division of Pathology, Department of Diagnosis and Therapeutic Sciences, Meikai University School of Dentistry, collected between 2000 and 2008, were used in this study.

\section{Immunohistochemical analysis}

Inflamed gingival tissues embedded in paraffin wax were sectioned and mounted on microscopic slide glass. Deparaffinized sections were immersed in absolute methanol containing $0.3 \% \mathrm{H}_{2} \mathrm{O}_{2}$ for $15 \mathrm{~min}$ at room temperature to block endogenous peroxidase activity. After washing, the sections were immersed in $0.01 \mathrm{M}$ citrate buffer, $\mathrm{pH} 6.0$, and heated in a microwave oven for

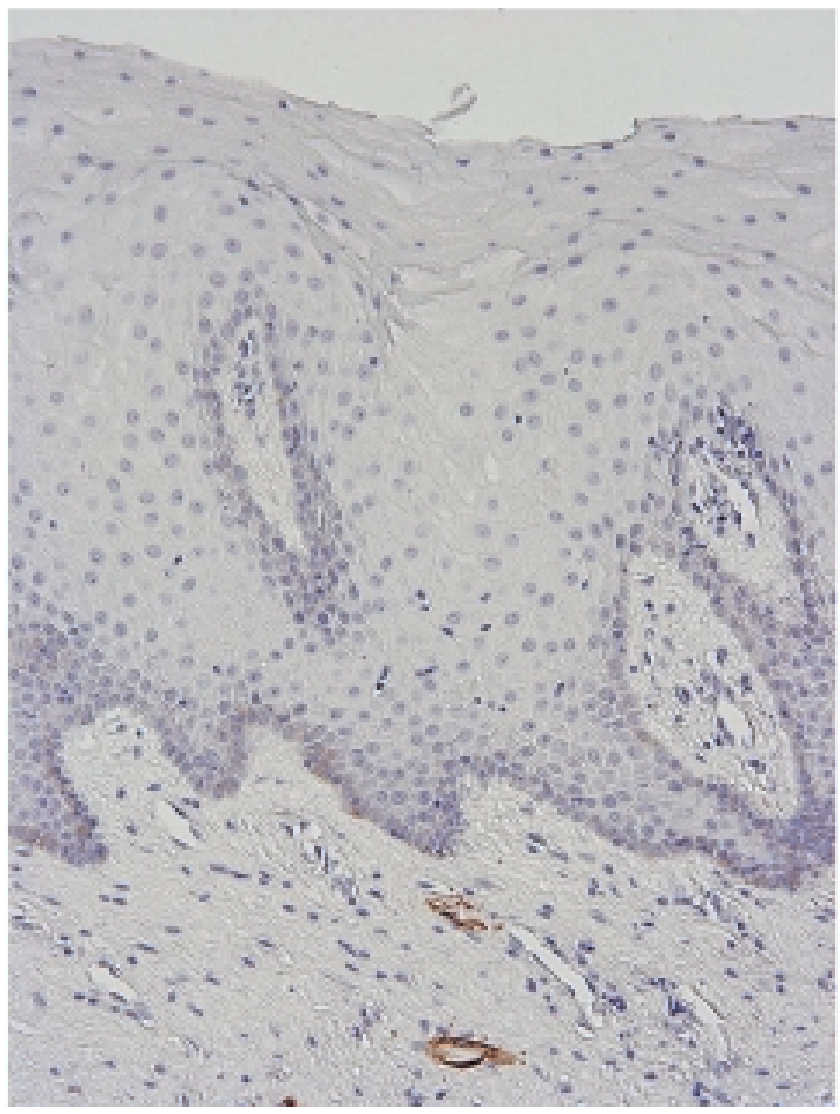

Fig. 1 Expression of podoplanin in the gingival tissue. A positive immunoreaction product for podoplanin was found in lymphatic endothelial cells in the connective tissue. Little or no reactivity was present in the oral epithelium.
5 min at high voltage and then for 15 min at low voltage. An appropriately diluted mouse monoclonal anti-human D2-40 (anti-podoplanin antibody; Dako North America, Inc., Carpinteria, CA) was applied to the sections for $1 \mathrm{~h}$ at room temperature, followed by a prediluted anti-mouse IgG antibody conjugated with peroxidase (Nichirei, Tokyo, Japan) was applied for $1 \mathrm{~h}$ at room temperature. The sections were immersed for $8 \mathrm{~min}$ in $0.05 \% 3,3^{\prime}$ diaminobenzidine tetrahydrochloride (DAB) in $0.05 \mathrm{M}$ Tris$\mathrm{HCl}$ buffer $(\mathrm{pH} 8.5)$ containing $0.01 \% \mathrm{H}_{2} \mathrm{O}_{2}$ and then counterstained with Mayer's haematoxylin.

\section{Results}

A positive immunoreaction product for podoplanin was found in lymphatic endothelial cells in the connective tissue of all inflamed gingival cases used in this study (Fig. 1). The basal cells of oral gingival epithelium showed positive immunoreactivity for podoplanin. The intensity was higher, when severe inflammatory cell infiltration was present in the connective tissue just under the

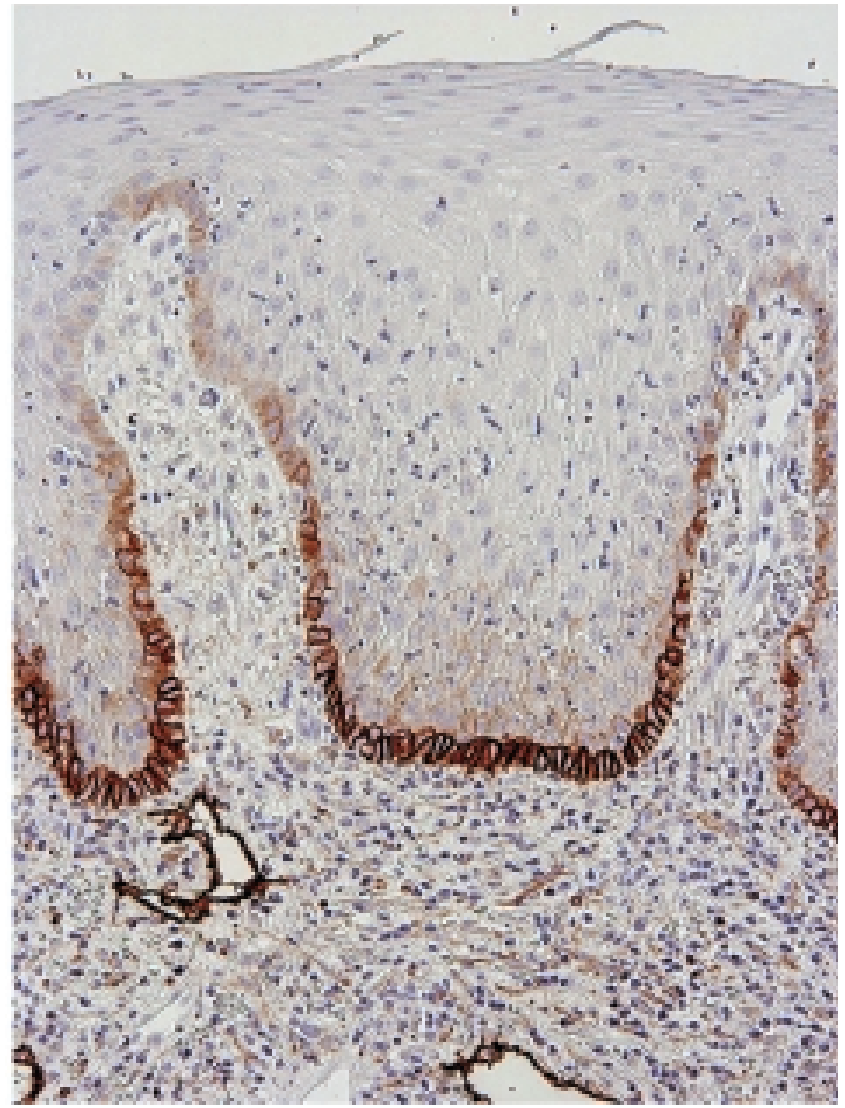

Fig. 2 Expression of podoplanin in the oral gingival epithelium. The basal cells showed a strong positivity for podoplanin. Severe inflammatory reaction was found in the connective tissue. 
epithelium (Fig. 2). When inflammatory changes were weak or absent, little or no reactivity for podoplanin in the basal cells was found (Fig. 1). Positive immunoreaction

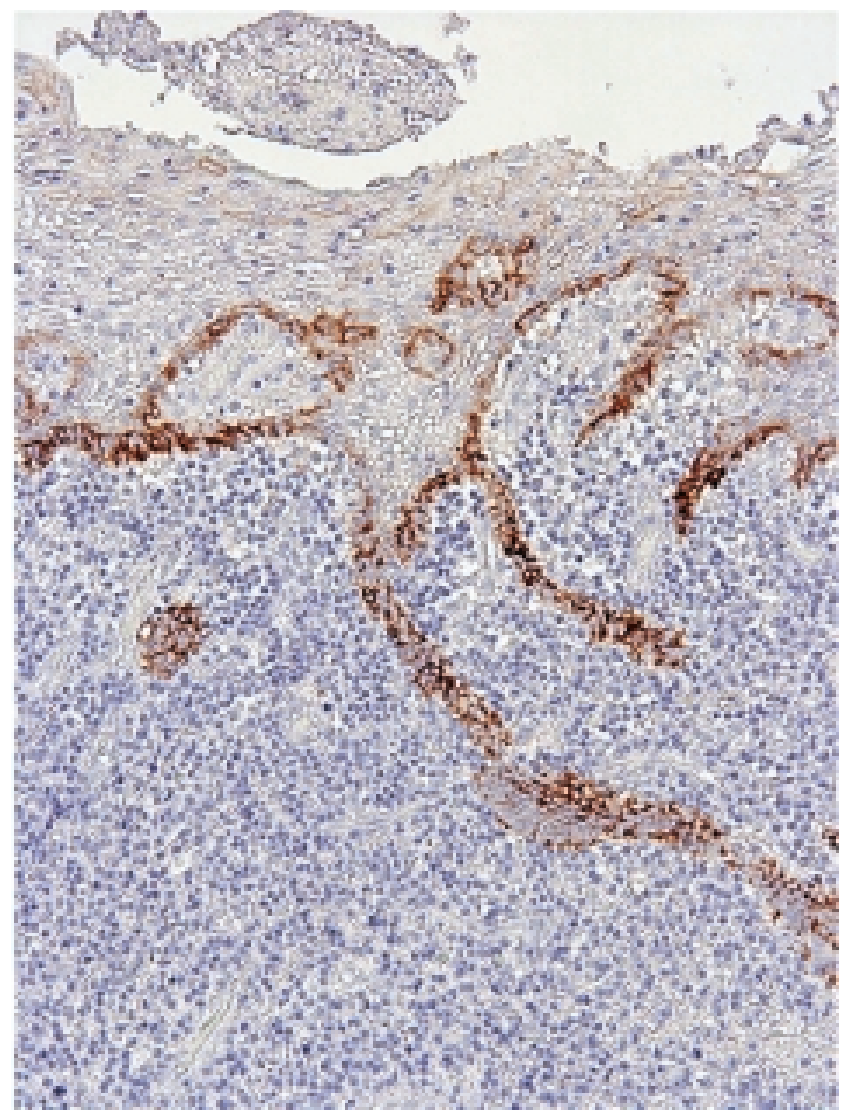

Fig. 3 Expression of podoplanin in basal cell extensions.
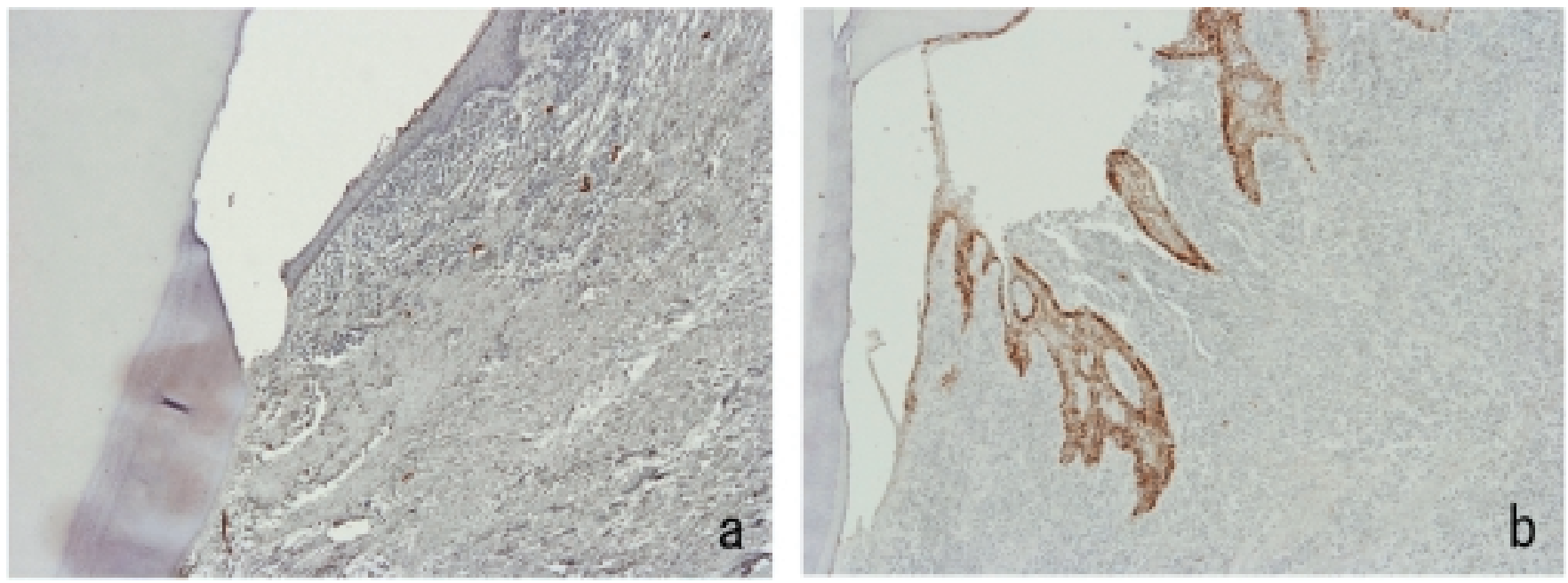

Fig. 4 Expression of podoplanin in the oral sulcular and junctional epithelia. Little or no positive reaction was detected in the product for podoplanin was also observed in basal cell extensions (Fig. 3). Little or no positive immunoreaction for podoplanin was detected in the oral sulcular and junctional epithelia without rete peg formation associated with slight inflammatory reaction in the connective tissue (Fig. 4a). However, strong positivity for podoplanin was observed in all layers of the oral sulcular and junctional epithelia including the elongated rete pegs associated with severe inflammatory reaction in the connective tissue (Fig. 4b). Podoplanin expression was detected in the cell membrane as well as in the cytoplasm of the epithelial cells.

\section{Discussion}

Human podoplanin is a $38 \mathrm{kD}$ type 1 transmembrane sialomucin-like glycoprotein consisting of 162 amino acids. Although the protein has been regarded as a specific marker for lymphatic endothelial cells (2,3,7-9), its expression has been reported in various normal as well as neoplastic cells $(4-7,10-15,20)$.

In normal cells other than lymphatic endothelial cells, focal expression of podoplanin has been detected on basal epithelial keratinocytes of the skin, cervix and esophagus $(2,3)$. On the other hand, no positive reaction for podoplanin has been demonstrated in basal cells of morphologically normal oral mucosal epithelium. However, its expression has been found in the cases where biopsy had been performed previously (10), suggesting that podoplanin expression may appear when morphologic changes such as regeneration or reparative processes occur. In addition to basal epithelial keratinocytes, high podoplanin expression has been detected on myoepithelial cells of the breast glands and salivary glands (3). We have also confirmed

\footnotetext{
epithelium without rete peg formation (a). Strong positivity for podoplanin was observed in all layers of the epithelium
with elongated rete pegs. Severe inflammatory reaction was found in the connective tissue just under the epithelium (b).

epithelium without rete peg formation (a). Strong positivity for podoplanin was observed in all layers of the epithelium
with elongated rete pegs. Severe inflammatory reaction was found in the connective tissue just under the epithelium (b).
} 
that podoplanin is expressed on myoepithelial cells of the major as well as minor salivary glands (20). Moreover, it has been reported that myofibroblasts of the prostate, fibromyocytes of the testis and perineural cells express podoplanin (3). Schacht et al. have pointed out that all of these cell types are contractile and undergo frequent changes in their shape mediated by myofilaments, and that podoplanin might have an important role in mediating cellular contractility and cytoskeletal reorganization (3). Other than these findings, expression of the glycoprotein has been found in alveolar type 1 cells of the lung, stromal reticular cells and follicular dendritic cells in the follicular germinal centers of lymphoid organs such as the thymus, tonsils and lymph nodes (16-19).

Increased expression of podoplanin has been detected in various malignant tumors, and a potential role of the glycoprotein in tumor progression has been suggested. However, we found that podoplanin is expressed in limited myoepithelial elements of pleomorphic adenomas (20). Although pleomorphic adenoma is a common benign epithelial tumor of the salivary glands, it has a tendency to invade surrounding tissues. In ovarian tumors, Schacht et al. have reported strong expression of podoplanin in the tumor cells of dysgerminoma and granulose cell tumor (3). However, other ovarian tumors such as adenocarcinomas, thecomas and Sertoli-Leydig cell tumors were negative for podoplanin $(3,21)$. Importantly, it has been described that the majority of primary squamous cell carcinomas are positive for podoplanin, and that there is an inverse relationship between its expression and tumor differentiation (3,10-14). Podoplanin-expressing cells are found at the invasive tumor front in human squamous cell carcinoma. It has been reported that podoplanin increases the activities of Rho GTPases, mainly RhoA, contributing to cytoskeletal reorganization, suggesting an important role of podoplanin in tumor invasion and metastasis (46).

In the present study, all the specimens of human inflamed gingival tissues showed a positive immunoreaction for podoplanin in lymphatic endothelial cells as well as limited epithelial elements. In oral gingival epithelium, reactivity for podoplanin was observed in the basal cells when severe inflammatory changes were present in the connective tissue just under the epithelium. In addition, positivity for podoplanin was also detected in basal cell extensions. It has been reported that basal cell extensions are frequently observed in gingival epithelium, but not in other types of oral epithelium, and it implies that the gingival epithelium may possess characteristics of odontogenic epithelium $(22,23)$. Enhanced expression of podoplanin was especially evident in oral sulcular and junctional epithelia including the elongated rete pegs associated with severe inflammatory reaction in the connective tissue.

These findings suggest that inflammatory stimuli from periodontopathic bacteria may induce podoplanin expression in the oral sulcular and junctional epithelia and increase the migratory capability of the epithelia essential for the progression of chronic periodontitis.

\section{References}

1. Pihlstrom BL, Michalowicz BS, Johnson NW (2005) Periodontal diseases. Lancet 366, 1809-1820.

2. Schacht V, Ramirez MI, Hong YK, Hirakawa S, Feng D, Harvey N, Williams M, Dvorak AM, Dvorak HF, Oliver G, Detmar M (2003) T1 $\alpha /$ podoplanin deficiency disrupts normal lymphatic vasculature formation and causes lymphedema. EMBO J 22, 3546-3556.

3. Schacht V, Dadras SS, Johnson LA, Jackson DG, Hong YK, Detmar M (2005) Up-regulation of the lymphatic marker podoplanin, a mucin-type transmembrane glycoprotein, in human squamous cell carcinomas and germ cell tumors. Am J Pathol 166, 913-921.

4. Martín-Villar E, Megías D, Castel S, Yurrita MM, Vilaró S, Quintanilla M (2006) Podoplanin binds ERM proteins to activate RhoA and promote epithelial-mesenchymal transition. J Cell Sci 119, 4541-4553.

5. Wicki A, Lehembre F, Wick N, Hantusch B, Kerjaschki D, Christofori G (2006) Tumor invasion in the absence of epithelial-mesenchymal transition: podoplanin-mediated remodeling of the actin cytoskeleton. Cancer Cell 9, 261-272.

6. Wicki A, Christfori G (2007) The potential role of podoplanin in tumor invasion. Br J Cancer 96, 1-5.

7. Wetterwald A, Hoffstetter W, Cecchini MG, Lanske B, Wagner C, Fleisch H, Atkinson M (1996) Characterization and cloning of the E11 antigen, a marker expressed by rat osteoblasts and osteocytes. Bone 18, 125-132.

8. Petrova TV, Mäkinen T, Mäkelä TP, Saarela J, Virtanen I, Ferrell RE, Finegold DN, Kerjaschki D, Ylä-Herttuala S, Alitalo K (2002) Lymphatic endothelial reprogramming of vascular endothelial cells by the Prox-1 homeobox transcription factor. EMBO J 21, 4593-4599.

9. Hirakawa S, Hong YK, Harvey N, Schacht V, Matsuda K, Libermann T, Detmar M (2003) Identification of vascular lineage-specific genes by transcriptional profiling of isolated blood vascular and lymphatic endothelial cells. Am J Pathol 162, 
575-586.

10. Dumoff KL, Chu CS, Harris EE, Holtz D, Xu X, Zhang PJ, Acs G (2006) Low podoplanin expression in pretreatment biopsy material predicts poor prognosis in advanced-stage squamous cell carcinoma of the uterine cervix treated by primary radiation. Mod Pathol 19, 708-716.

11. Kato Y, Kaneko M, Sata M, Fujita N, Tsuruo T, Osawa M (2005) Enhanced expression of Aggrus (T1alpha/podoplanin), a platelet-aggregationinducing factor in lung squamous cell carcinoma. Tumor Biol 26, 195-200.

12. Martín-Villar E, Scholl FG, Gamallo C, Yurrita MM, Muñoz-Guerra M, Cruces J, Quintanilla M (2005) Characterization of human PA2.26 antigen (T1alpha-2, podoplanin), a small membrane mucin induced in oral squamous cell carcinomas. Int $\mathrm{J}$ Cancer 113, 899-910.

13. Yuan P, Temam S, El-Naggar A, Zhou X, Liu DD, Lee JJ, Mao L (2006) Overexpression of podoplanin in oral cancer and its association with poor clinical outcome. Cancer 107, 563-569.

14. Nakayama Y, Matsumoto K, Nagato M, Inoue Y, Katsuki T, Minagawa N, Shibao K, Tsurudome Y, Hirata K, Higure A, Sako T, Nagata N (2007) Significance of lymphangiogenesis as assessed by immunohistochemistry for podoplanin in patients with esophageal carcinoma. Anticancer Res 27, 619-625.

15. Scholl FG, Gamallo C, Vilaró S, Quintanilla M (1999) Identification of PA2.26 antigen as a novel cell-surface mucin-type glycoprotein that induces plasma membrane extensions and increased motility in keratinocytes. J Cell Sci 112, 4601-4613.

16. Dobbs LG, Williams MC, Gonzalez R (1998) Monoclonal antibodies specific to apical surfaces of rat alveolar type I cells bind to surfaces of cultured, but not freshly isolated, type II cells. Biochim Biophys Acta 970, 146-156.

17. Rishi AK, Joyce-Brady M, Fisher J, Dobbs LG, Floros J, VanderSpek J, Brody JS, Williams MC (1995) Cloning, characterization, and developmental expression of a rat lung alveolar type I cell gene in embryonic endodermal and neural derivatives. Dev Biol 167, 294-306.

18. Williams MC, Cao Y, Hinds A, Rishi AK, Wetterwald A (1996) T1 alpha protein is developmentally regulaterd and expressed by alveolar type I cells, choroid plexus, and ciliary epithelia of adult rats. Am J Respir Cell Mol Biol 14, 577-585.

19. Ramirez MI, Millien G, Hinds A, Cao YX, Seldin DC, Williams MC (2003) T1 $\alpha$, a lung type I cell differentiation gene, is required for normal lung cell proliferation and alveolus formation at birth. Dev Biol 256, 61-72.

20. Oku Y, Tanaka A, González-Alva P, Sakashita H, Kusama K (2008) Podoplanin expression in human pleomorphic adenomas. In: Oral oncology, Vol 12, Varma AK, Qiu WL eds, Ocean Papers \& Printers, Shanghai, 251-253.

21. Kawase A, Ishii G, Nagai K, Ito T, Nagano T, Murata Y, Hishida T, Nishimura M, Yoshida J, Suzuki K, Ochiai A (2008) Podoplanin expression by cancer associated fibroblasts predicts poor prognosis of lung adenocarcinoma. Int $\mathrm{J}$ Cancer 123, 1053-1059.

22. King RE, Altini M, Shear M (1979) Basal cell extensions in human oral mucosa. J Oral Pathol 8, 140-146.

23. Tanaka A (2004) Studies on basal cell extensions in the human gingival epithelium. Meikai Daigaku Shigaku Zasshi 33, 182-192. (in Japanese) 